\title{
The Effect of Inattention on Form Perception
}

\author{
Irvin Rock and Daniel Gutman \\ Institute for Cognitive Studies, Rutgers-The State University
}

\begin{abstract}
A state of inattention was achieved by having subjects selectively attend to one of two overlapping novel figures in a series of such overlapping figures. Recognition of form directly afterward was good for figures that had been attended to but was essentially nil for the unattended figures. Recognition failed to occur even if a familiar figure was in the unattended series and even if that figure was presented $1 \mathrm{sec}$ before the test. A further experiment showed that certain general characteristics of the unattended figures other than form were recognized. The results are interpreted as indicating that attention is necessary for form perception, not merely for memory of form. It is suggested that a cognitive process of description constitutes the essence of form perception. Diverting attention eliminates the cognitive operation of describing the spatial relations that characterize a figure.
\end{abstract}

Suppose that one is looking directly at a figure but not attending to it. An example would be the situation that prevails in a wallpapered room where the eyes must often fixate on one or another representation of the repeated pattern when the observer is not attending to it. Is the shape of the pattern perceived? There undoubtedly is an awareness that some pattern is there on the wall, but it is difficult to determine from such everyday life examples whether form perception entailing awareness of specific shape does or does not require attention.

However, it is possible to create experimental conditions in the laboratory in which an observer is looking directly at a figure without attending to it. In the method employed here, the observer is asked to view two overlapping figures of different colors (Figure 1) and is given a task that requires attention to the figure of one color. The subject is to maintain fixation on a region lo-

This work was supported in part by Grant MH 30865 from the National Institute of Mental Health to Rutgers University, Irvin Rock principal investigator.

We thank Joseph Di Vita for his help in obtaining eye movement recordings and John Ceraso, Judith Kroll, Robert Schindler, and Deborah Wheeler for their advice concerning analysis of the data and the interpretation of the findings.

Requests for reprints should be sent to Irvin Rock, Institute for Cognitive Studies, Rutgers-The State University, Newark College, Newark, New Jersey 07102. cated in the center of the two figures. Thus, in principle each figure stimulates the central retina equally, but one is attended to and the other is not. Following the presentation of these overlapping figures, recognition of each figure is tested.

Any superiority in the subsequent recognition of the attended figure over the unattended one would thus presumably result from attention versus inattention. Although the question of a difference between attending or not attending to a figure is of interest in relation to the matter of form perception, perhaps the important question here is whether there is any significant perception (and thus subsequent recognition) at all of the shape of the unattended figure. With respect to the former question of a difference, it is important to be sure that there is no advantage for the attended figure resulting from a difference in the retinal locus of that figure based on eye position or eye movement as compared to that of the unattended one. For if there were, the difference could be attributable to eye position or eye movement rather than to attention per se. In this regard it is important to note that even if fixation is not perfectly maintained, the overlapping of figures is such that shifts in eye position would not necessarily give an undue advantage to the attended figure as far as the retinal location of contours is concerned. 

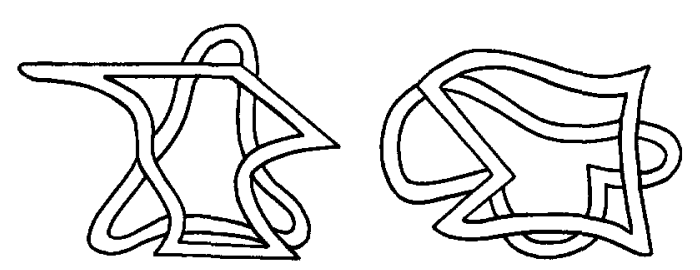

Figure 1, Examples of the figures used in Experiment 1. (The overlapping figures were colored red and green within the outlines shown here.)

With respect to the question of whether there is any perception of the unattended figure, the possibility of some slight advantage for the attended figure based on its retinal locus as a function of eye position is not altogether crucial. The fact is that with attention, the visual angle subtended by either of the overlapping figures in the experiments to be reported is small enough to enable the figures' shape to be perceived adequately either without any change of fixation, as in a brief tachistoscopic exposure, or regardless of what region or regions in the overlapping pattern are fixated.

A further question about the design of this kind of experiment is: Precisely what is meant by "perception?" Obviously, some properties of the unattended figure will be perceived. At a minimum its color must be perceived, and, since it is the contour that embodies the color, there must be some perception of the contour of the unattended figure. Clearly, then, the issue is whether or not an aspect of the unattended object, namely, its specific shape, is apprehended, not whether certain other of the object's properties are or are not detected. The first four experiments reported below focus on this question of form or shape perception, but in the final experiment an attempt is made to explore the question of what properties of the unattended figure other than shape are perceived.

There is also the central question of whether the effect of inattention on form concerns failure of perception or of memory. The test employed in these experiments is recognition so that failure can mean either some inadequacy in the initial perception or in the retention of the product of that perception. In what follows, this question is addressed, but it is acknowledged in advance that we will not present evidence that can be taken as unequivocal in support of our belief that the effect of inattention in this kind of experiment is on perception. We will, however, present evidence and argument that makes that interpretation the more plausible one.

\section{Experiment 1}

\section{Method}

Subjects. The subjects were 20 Rutgers male and female undergraduates, 10 attending to red figures and 10 attending to green figures. An additional 10 subjects served in a separate condition in which eye movements were monitored. In this and the remaining experiments, all subjects were naive about the purpose of the experiments. No subject participated in more than one of these experiments, and none of the subjects was color blind.

Procedure. Subjects were told that the experiment was concerned with aesthetic judgment under conditions of distraction. A Hunter Model 340 Cardmaster presented a $61 / 4$ in. $\times 31 / 2$ in. $(15.9 \mathrm{~cm} \times 8.9 \mathrm{~cm})$ white card for $1 \mathrm{sec}$, with $2 \mathrm{sec}$ between successive cards. There was normal room lighting. Subjects were instructed to fixate on a black dot placed on the glass in front of the window and located in the center of the window so as to appear in the central region of each pair of overlapping figures. On each card were two outlines of novel forms, equivalent in style and approximately equivalent in complexity. An example is shown in Figure 1. One of the outlines was red, and the other was green. All figures were about $3 \mathrm{in} .(7.6 \mathrm{~cm})$ high and wide (subtending a visual angle of about $31 / 2^{\circ}$ ), and the lines were $1 / 8$-in. $(.32 \mathrm{~cm})$ thick. The figures were cut out of colored construction paper and glued to the cards, one figure right on top of the other, but occluding as little of the bottom figure as possible. Half of the time the red figure was on top, and half of the time the green figure was on top. The subjects sat $4 \mathrm{ft}$. $(1.22 \mathrm{~m})$ from the Cardmaster, with its window at eye level.

The subjects were first shown a sample card and were told that they would be asked to rate the red (or green) figures on a scale from one to five according to how pleasing they were and that the cards would be presented in rapid succession. The subjects were then shown a practice trial with five cards to acquaint them with the task and to make certain that they understood the instructions. The experimenter recorded these subjects' ratings as well as those of the main series of cards. The colored figures that the subjects were not rating were called the distraction figures and will be referred to here as the unattended figures.

Ten cards were then presented, and directly afterward a recognition test sheet was presented. The sheet contained 15 black figure outlines, 5 from the middle of the attended series, 5 from the middle of the unattended series, and 5 new ones, in random positions. Therefore, the subjects were presented with 10 of the previously exposed figures, although they had attended to only 5 of them. They were asked to encircle any of the figures 
on the sheet that they recognized from the series they had just seen, regardless of what color the figures had been. No time limit was imposed.

Since it might be argued that subjects did not maintain fixation perfectly in the above procedure, a further condition was included in which eye position was recorded. We were interested in ascertaining just how adequately subjects did maintain fixation. Moreover, despite what was said above about this question, it might be maintained that within the 1-sec exposure of each card, subjects could selectively move their eyes saccadically to fixate several points on the contour of the attended figure rather than on the unattended figure. Such movements might be said to favor the attended figure, although, as noted above, it could not be said to eliminate the possibility of perceiving the unattended one. To investigate this question, a separate group of 10 subjects was shown the same series of cards and instructed to rate the green figures while fixating the central dot. Their eye movements were recorded by means of the Eye View Monitor, Model 1992S, manufactured by $G$ and W Applied Science Laboratories (previously known as the Whittaker Eye View Monitor). The figures were not presented on cards, however, but as slides by a projection tachistoscope, in the manner employed and described in Experiment 4. Data from a few subjects were discarded because adequate eye recording could not be obtained. Aesthetic "ratings" were reported by appropriate finger movements, since talking would have interfered with the eye recording.

\section{Results}

Of a mean of 4.9 of the 15 figures on the recognition test sheet that were circled, 3.2 were figures the subjects had been attending to, .95 were unattended figures, and .75 were figures that had never been presented. The results were similar for red-attending and green-attending subjects. The mean for attended figures was significantly different from the mean for unattended figures, $t(19)=5.9, p<.001$, and from the mean for new figures, $t(19)=8.1, p<.001$. The mean for unattended and the mean for new figures were not significantly different from each other $(t=.75)$. Thus, recognition of the attended figures occurs, but since subjects did not choose significantly less of the figures that they had never seen than they did figures seen but not attended to, it would seem that there was no recognition of the unattended figures.

A possible criticism of this experiment, however, is that subjects set a high criterion of certainty for themselves in identifying figures to which they had not attended. Thus, they may have avoided selecting figures on the test that were in the unattended series when they did not feel certain. To test this possibility, immediately following the recognition test as described above, a forcedchoice procedure was added. The subjects were told that in fact there were 10 figures on the sheet that they had seen and that since they had just circled less than that number, they were now to try to pick out the remaining ones by checking them. When the data for the initial and this forced-choice test were combined, the mean numbers of selections were $4.55,3.05$, and 2.45 for the attended, unattended, and new figures, respectively.

These means no longer reflect the great superiority of the attended figures relative to the others obtained in the first test, but it must be understood that in the pool of uncircled figures from which the subjects were choosing, there were fewer attended figures available. By and large the subjects felt that they were guessing in the forced choice. Such guessing would tend to water down any superiority of attended figures, particularly if most of those that were recognizable had already been circled. In any event, the mean for the attended figures differs significantly from both of the other means, $t(19)=4.36$ and $7.29, p<.001$, but the mean for the unattended figures does not differ significantly from the mean of the new figures, $t(19)=1.29$. Although there is some increase in the difference between unattended and new figures as a result of the forced-choice test, it is not sufficient to establish that significant recognition of the unattended figures occurred. Of course, the null hypothesis cannot be proven, and it remains possible that by some more sensitive method of testing such recognition would be evidenced.

The results of the eye-movement recording condition were as follows: Out of a mean of 4.2 of the 15 figures on the recognition sheet that were circled, 2.4 were figures that the subjects had been attending to, .80 were unattended figures, and 1.0 were new figures that had not been presented. (The forcedchoice procedure was not used in this condition.) The mean for attended figures is significantly greater than the mean for unattended figures, $t(9)=2.85, p<.02$, and significantly greater than the mean for new 
figures, $t(9)=3.5, p<.01$. The means for unattended and new figures were not significantly different from each other, and, in fact, the difference favors the new figures. Thus, the quantitative results correspond closely with those of the unmonitored condition reported above. The somewhat lower recognition of the attended figures here may be based on the shorter interfigure interval during exposure of the slide series-1 sec rather than 2-used in this condition, or it may have resulted from the distraction and slight fatiguing created by the process of calibrating eye position prior to presenting the figures.

As to eye movements, the recordings for these 10 subjects revealed little in the way of excursion from the fixation point throughout the viewing of all 10 figures. Few, if any, of the 100 separate recordings (10 subjects $\times 10$ figures each) indicated any distinct saccadic movements to regions of the figure remote from the fixation point. In most cases, the recording showed only slight oscillations or drifts of the order of no more than $1^{\circ}$ centered either exactly on the fixation point or on a region no more than $1^{\circ}$ from it, and these movements did not favor contours of the attended over the unattended figures. Thus, it is not evident from these recordings how any advantage for the attended figures would result from whatever failure there was to maintain precise fixation on the dot. More important, it is difficult to argue that adequate perception of the unattended figure would in any way be prevented by the locus of its retinal image as determined by these recordings.

\section{Experiment 2}

It is clear from Experiment 1 just how important attention is for recognition of form. But we cannot say from the results what effect, if any, the unattended figures had on the recognition of the attended figures. Although the unattended figures were by and large not recognized, perhaps they interfered with the processing of the attended figures. Without such interference the number correct might have been closer to five.

\section{Method}

Subjects. The subjects were 20 male and female Rutgers undergraduates.

Procedure. In Experiment 2, instead of having a red figure and a green figure overlapping on a card, the subjects were presented with 10 cards with only one figure on each, all red for half of the subjects and all green for the other half. The two figures on the cards from Experiment 1 were simply peeled off and placed on two separate cards for Experiment 2. The procedure and instructions were otherwise the same, except that reference to distraction was deleted.

After viewing the 10 cards, subjects were given the same recognition test sheet as was used in Experiment 1 and were asked to circle any figures that they recognized. In this experiment, only 5 of the 15 figures on the page had been seen.

\section{Results}

There was no difference in results for subjects shown the red and those shown the green figures. The subjects circled a mean of 4.75 figures on the page. Of these, 3.6 were figures they had seen and 1.15 were new, a difference that is highly significant, $t(19)=7.3, p<.001$. To compare recognition here with that of attended figures in Experiment 1, the correct recognitions for each subject were expressed as percentages of the total number of figures circled. The mean percentage of correctly circled figures in this experiment is 75.3 , and this does not differ significantly from that of 70.2 obtained in Experiment 1 for correctly circled attended figures under otherwise comparable conditions, so it is concluded that not only are the unattended figures not perceived, but they also do not seem to interfere with the processing of the attended forms.

\section{Experiment 3}

It might be argued that even though the unattended figures in Experiment 1 were not recognized in the test, this does not mean that they were not perceived. Perhaps they were perceived and forgotten by the time the test was given. With that in mind, Experiment 3 introduced two familiar figures into the series, with the assumption that familiar figures, if perceived, would less likely be forgotten than novel forms. They would enjoy the further advantage of isolation in this procedure, since there are only 2 in the series of 10 cards. 


\section{Method}

Subjects. The subjects were 20 male and female Rutgers undergraduates.

Procedure. The same cards and procedure of Experiment 1 were used, except that on the fifth card, the red novel form was peeled off and replaced with a red Christmas tree shape, and on the eighth card, the green novel form was peeled off and replaced with a green outline of a house. Both familiar forms were glued on top of the novel forms with which they were paired (Figure 2). Therefore, red-attending subjects had a tree among their attended figures and a house among the unattended ones, and green-attending subjects had a house among their attended figures and a tree among the unattended ones. If the unattended figures had been perceived, we would expect that they would immediately be recognized, so that all subjects would know that there was both a tree and a house presented to them. If the unattended figures had not been perceived, we would expect the red-attending subjects to know that there was a tree but not to know that there was a house, and the green-attending subjects to know there was a house but not a tree.

To control for order of the familiar figures, Cards 5 and 8 were switched in the series for half of the subjects. There were 3 practice cards. After viewing the 10 cards, the subjects were immediately asked, "Did you see any familiar figures in the series you were just shown?" When they had responded and their responses were recorded, if they had not responded with both "house" and "Christmas tree," they were asked if they had seen the familiar figure that they had not mentioned. Subjects were then briefly interviewed and, in doubtful cases, were asked to sketch one or both familiar figures.

\section{Results}

By and large, the subjects recognized the familiar figure that they were attending to but were unaware of the unattended familiar figure. Seventeen of the subjects, or $85 \%$, reported the familiar attended figure and 1 more claimed to have seen it when told what it was. Only 2 subjects, or $10 \%$, reported the
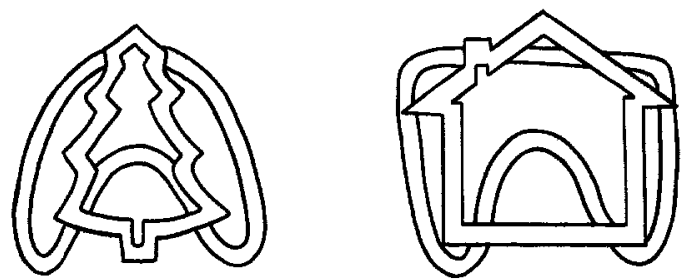

Figure 2. The two familiar figures used in Experiments 3 and 4 shown with the overlapping novel figure with which each was paired. (The overlapping figures were colored red and green within the outlines shown here.) familiar unattended figure, although 3 more claimed that they had seen it after they were told what it was. However, 1 of the 2 was clearly not following instructions, and 2 of the 3 drew pictures that did not correspond at all with the kind of house or tree actually presented. One can thus reasonably conclude that the few reports of the unattended familiar figure are either spurious or are explicable in terms of "disobedient" fluctuations of attention.

Apparently the unattended familiar figure was not perceived. This provides evidence that the poor recognition performance with the unattended figures in Experiment 1 was not simply a failure of memory.

\section{Experiment 4}

It might still be argued that the assumption that familiar figures will not be forgotten is incorrect. A method was therefore devised to test the subject's knowledge of the unattended familiar figure immediately after it had been presented.

\section{Method}

Subjects. The subjects were 20 male and female Rutgers undergraduates.

Procedure. In Experiment 4, the cards with familiar figures were followed by a blank card, and the subjects were given instructions beforehand to the effect that if a blank card should ever appear, they were to describe in a few words whatever they saw just before the blank.

Slides were made of the stimulus cards used in the previous experiment and projected on a 12 in. $\times 18$ in. $(30.5 \mathrm{~cm} \times 45.7 \mathrm{~cm})$ white screen with a fixation dot in the middle by a Lafayette Instrument Company Model 42011 two-channel tachistoscope. The size of the projected figures was 6 in. $(15.2 \mathrm{~cm})$. The tachistoscope was placed $7 \mathrm{ft}$. $(2.13 \mathrm{~m})$ away from the screen, and the subjects sat $8 \mathrm{ft}$. ( $2.44 \mathrm{~m})$ away from the screen so that the figures subtended the same visual angle as in the previous experiments. The tachistoscope exposed each slide for $1 \mathrm{sec}$, and the interval between slides was $1 \mathrm{sec}$. Experiment 4 took place in the dark.

Subjects were given the same preliminary instructions as in Experiment 1 and were shown 5 practice slides. They were then given the special instructions concerning blank cards. In the series of 10 slides, the familiar-figure cards appeared in Positions 3 and 9 and the blanks at 4 and 10. Each of the other six positions had a slide with two novel forms that had been used in Experiment 3. For the red-attending subjects, the slide with the Christmas tree was in the third position, and the one with the house was in the ninth position. For the greenattending subjects, the house was in the third position 
and the tree was in the ninth position. In this way, the slide prior to the last blank had the unattended familiar figure on it for all subjects. After the last blank, the tachistoscope was immediately turned off, and subjects had ample time to describe whatever they had seen before the blank. Their responses were tape recorded.

\section{Results}

Eighty percent of the subjects called out the familiar figure they were attending to when the first blank appeared, but when the second blank appeared, they described the novel form to which they were attending and did not mention the unattended familiar figure. When they were subsequently probed about that unattended figure, none of them was aware that there was a familiar figure there. When shown the card with the familiar figure in the interview, these subjects said that they had not seen it.

Of the four subjects who did not respond in this way, one of them claimed in the posttest interview that instead of rating the red figures as instructed, he had been rating the red figures in comparison to the green figures. Thus, this subject did not follow instructions. Another one of these four was totally confused by the experiment and saw no familiar figures at all. If these two subjects are omitted, $89 \%$ of the subjects were aware of the familiar figure they were attending to but were unaware of the one they were not attending to. These results are comparable to those of Experiment 3.

Since most of the subjects did not report the second familiar figure 1 sec after it had been presented, it seems unlikely that the failure to report the unattended familiar figures in Experiment 3 was due to a failure of memory. They apparently were not perceived.

\section{Experiment 5}

Although the evidence is thus strong that the shape of a figure is not perceived when not attended to, perhaps other characteristics of the figure are perceived. For example, we can safely assume that the color of the unattended figure is perceived. In the following experiment, we investigate this question by testing for recognition of properties other than form itself. We were interested in whether or not features such as size, mode of contour line, the straightness or curvature of the contour lines, and the open or closed character of the figure would be recognized in a test directly after exposure of the unattended figure displaying one or another of these features.

\section{Method}

Subjects. The subjects were 80 Rutgers male and female undergraduates, 20 in each of four conditions.

Procedure. All of the subjects attended to red, and after receiving the same preliminary instructions as in Experiment 1 and viewing five practice slides, they were shown four slides. The first and third slide in each condition were simply red and green rectilinear closed, same-size, same-mode figures paired together. In Slides 2 and 4 , though, the red figure was similar to those of Slides 1 and 3 , but the unattended green figure was different. In Condition 1, the green figure was curvilinear rather than rectilinear in both of these slides. In Condition 2, the green figure was very large (or, for half of the subjects, very small) in Slide 2 and very small (or very large) in Slide 4 . In Condition 3, the green figure was open rather than closed in both these slides, open to the side in Slide 2 and open on top in the Slide 4 (or vice versa for half of the subjects). In Condition 4 , the green figure was made of dots in Slide 2 and dashes in Slide 4 (or vice versa for half of the subjects).

The purpose of introducing the relevant kind of new feature in the unattended figure of the second card in addition to doing so in the critical figure of the fourth card was to attempt to prevent attention from shifting during presentation of the fourth card. The introduction of the novel feature only on the critical fourth card might otherwise have drawn the subject's attention. If so the subject's state of mind with regard to the critical green figure would not be entirely the same as in the previous experiments described here, namely, one of inattention.

After viewing the four slides, subjects were tested to see what, if anything, they knew about the unattended green figure in the last slide. In Condition 1 , they were simply asked if that figure had been made up of straight lines or curved lines. In Condition 2, they were shown a slide of that figure outlined in three different sizes and were asked to indicate which size it had been. For half of the subjects the larger figure was on the right and the smaller one on the left in this test slide, and for the other half, the reverse was the case. In Condition 3, they were simply asked if the figure had been open or closed. In Condition 4, they were shown a slide with four different modes of contour line on it, and they had to choose which mode had been the same as the unattended green figure. The four modes were a continuous-line contour, a dotted-line contour, a dashed-line contour, and a contour composed of small xs. For half of the subjects, the test slide was in one orientation, and for the other half, it was in the inverted orientation, thus changing the topto-bottom location of the four alternative modes. 


\section{Results}

Of the 20 subjects in Condition 1,11 were correct and 9 incorrect in their description of the critical green figure as composed of curvilinear or rectilinear contours, respectively, a result obviously not different from the chance expected values of 10 in each case. Of the 20 subjects in Condition 2, 18 correctly selected the larger or smaller figure in the test slide, a result that is significantly different from chance which, based on random guessing, would have been $6.67, \chi^{2}(2)=$ $28.8, p<.01$. Of the 20 subjects in Condition 3,15 correctly indicated that the critical figure had been open, and 5 incorrectly indicated that it had been closed, a result significantly different from the chance expected values of 10 in each case, $\chi^{2}(1)=5, p<.05$. Of the 20 subjects in Condition 4, 15 selected the correct contour mode (dashed or dotted), and 5 selected an incorrect mode, a result significantly different from chance, which, based on random guessing, would have been 5 and 15 , respectively, $\chi^{2}(1)=26.7, p<.01$. Moreover, the five incorrect choices were of the mode that was the one used for the unattended green figures exposed on the second slide.

These results indicate that features such as size, type of figure (open or closed), and mode of contour of unattended figures are often perceived despite the failure to perceive the shape of the figure. The fact that the straight or curved nature of the contours does not seem to be noted suggests that this feature is a more integral part of the shape than the other features tested, at least in the case of the method employed here.

\section{Discussion}

The results of this series of experiments are confirmed by those of a prior study on the same problem but using an entirely different method (Rock, Schauer, \& Halper, 1976). In Rock et al. a state of inattention was created without the use of distracting or competing figures. The subjects believed that the experiment was concerned with the maintenance of an afterimage of a simple geometric shape exposed at the outset. A series of paper surfaces was then presented, each of which the subjects believed to be a "screen" they were to view and on which they were to report the presence or absence of an afterimage. The surfaces varied in color or texture, but each contained a fixation mark centered within an outline square. The last one in the series contained a novel geometrical shape within the outline square. By that time few if any subjects any longer experienced afterimagery. Therefore, the method entailed viewing a figure without attention. In a test given directly afterward containing that figure and one or more others of different shape, there was no evidence of beyond-chance recognition of the figure, provided the alternative figures in the test were of the same kind, size, level of complexity, and rectilinear contour. With only a slight change in the procedure so that the figure was viewed with attention-although incidentally, that is, without any intention to learn or remember it-recognition was good.

One problem with this method, however, is that there is little yield of data per subject, namely, only one item scored as either "correct" or "incorrect." Another problem is that the method rests on the rather subtle understanding on the part of the subjects concerning what they are doing. For the method to be successful, the subject must fully believe that the critical stimulus at the end of the exposure series is little more than one more surface for viewing their afterimage. We were, therefore, not certain that other investigators would have been able to duplicate the experiment.

In this earlier study, an experiment was also directed at the question of the perception of properties other than shape per se, analogous to Experiment 5 in the present series. The initial presentation of the critical figure was the same as described above, namely, the subjects believed that they were viewing it as one of a series of "screens" on which to project their afterimage. But in the test the subjects had to choose from among several alternatives all of the same shape but differing in either contour mode, size, closed or openness of figure, rectilinearity or curvilinearity of contour, and complexity of contour. For each of these conditions, beyondchance recognition occurred. Thus, these results confirm those of the present Experi- 
ment 5 except that they also show successful recognition of the straight or curved character of the figure's contour. We cannot explain the different results of the two methods concerning this feature but call attention to the fact that with one method the contours of the unattended figure are overlapped by other contours, and with the other method they stand alone.

The findings from both kinds of experiments indicating that although shape is not recognized without attention, other figural properties are is certainly of great interest and requires explanation. Clearly, form perception cannot be understood merely in terms of the registration and transmission of information concerning the physical contours or boundaries of a figure. We can safely assume that such processes occur with or without attention. In other words, iconic representation of both of the overlapping figures undoubtedly occurs. But phenomenal shape entails an apprehension by the observer of the exact spatial interrelationships of the parts of the figure to one another and of the relationships of these parts to the updown, left-right spatial coordinates. It is this apprehension that requires higher level processing culminating in a cognitive description of the specific shape of the figure as a unique, organized whole. It is this processing that requires focal attention. To put it differently, perhaps what attention amounts to is the process of engaging in such a cognitive act. Thus, it is the failure to engage in such descriptive processing of our unattended figure that is responsible for the failure to perceive it. Similar arguments have been presented by Hochberg (1970) and Neisser (1967). Other lines of evidence support the contention that perception is the result of a process of description (see Bregman, 1977; Reed, 1974; Rock, 1973, 1975; Rock \& Gilchrist, 1975; Rock \& Halper, 1969; Rock, Halper, \& Clayton, 1972; Rock \& Sigman, 1973).

Features other than shape, such as color, mode, size, and so forth, do not require this kind of processing and thus can be perceived even while the observer is directing his or her attention elsewhere. To be sure, the perception of these features must also require some kind of processing beyond that which leads to iconic representation. It would have to be both local-leading to the perception of properties such as contour mode and color-and global-leading to the perception of properties such as size, complexity, and so forth.

How should our findings be interpreted in the light of the vast literature on the problem of attention? It is difficult to compare our results with any of those reported by others because of a combination of features in our method that makes it unique. Thus, although the use of two simultaneous series of items, with attention directed to one, is similar to the dichotic-listening paradigm (Broadbent, 1958; Cherry, 1953; Treisman, 1969), our task concerns the processing of visual form rather than auditory meaning. Our method of achieving selective attention does not entail the requirement of repeating the attended item (shadowing) and is based on a feature of the stimulus items (color) rather than a "channel" distinguished by ear or location or some different kind of feature. Although other experiments on attention have utilized visual items (e.g., Shiffrin \& Schneider, 1977; Sperling, 1960), they were concerned with processing at the level of recognition, rather than with form per se. Moreover, whereas these other experiments sought to determine how many items can be apprehended with divided attention to peripherally presented items (see also Eriksen \& Lappin, 1967), our method investigates the effect of focused attention with both items presented to central vision.

This last difference is worth emphasizing. We do not maintain that focused attention is always necessary for form perception. Thus, for example, in reading we must be capable of identifying many of the letters that constitute the several words we can grasp in one glance. This implies the capability of simultaneous perception of the shape of many items. Similarly, in the studies referred to above, it has been demonstrated that more than one figure presented simultaneously by tachistoscope can be perceived. However, these effects do not entail attention directed to one item along with the necessary withdrawal of attention from another item because of the kind of task assigned, as does our experiment. Rather, they 
permit or require attention to multiple items. We would expect that with instructions to attend to both of our overlapping figures, recognition of both would have been reasonably good. The question of how widely attention can be effectively distributed is important but is not one we have addressed. To be sure, one would think that if form perception is based on description, there would be limits to how many separate items can be processed in a limited period. Thus, it is probable that the capacity to process multiple items simultaneously in reading is based on certain additional factors such as the organization of letters into higher order units (words and phrases), in which context, overlearning, and special conditions of practice enter in. We still have little understanding of how this works.

There are, however, cases in which attention is directed away from an unattended item, but it is nonetheless perceived. In these cases, that item is particularly salient, as in the well-known example using a dichotic-listening paradigm where the item is the subject's name (Moray, 1959). It is probable that an analogous effect could be produced with our paradigm of overlapping figures. The only way we can understand this kind of effect is to say that attention is drawn to the item. But why should that occur if it is not yet perceived? We would think that there must be detection of some of the sensory properties of the unattended item that suffice to suggest to the perceptual system what that item might be. This is a problem that needs to be resolved.

Based on other research reported in the literature, it might be argued that our methods of recognition and immediate recall are not sufficiently sensitive to reveal all that is cognized about the unattended figure. Thus, it has been claimed that the meaning of the unattended stimulus item (a word) can produce certain effects, such as altering the latency of shadowing of the simultaneously presented attended word (Lewis, 1970) or yielding conditioned galvanic skin responses to the unattended word (Corteen \& Wood, 1972). These measures are presumably sensitive to processing on a level that would have to include semantic content, but it is reported that there is no direct awareness or immediate recall of the item. Even if such effects are genuine, it does not necessarily follow that analogous effects would be demonstrable for form perception, with which we are here concerned. That is, they may only occur when meaning enters into the processing. But in the light of the earlier history on so-called "subception," perceptual defense or subliminal effects in which artifacts and alternative explanations were often uncovered, we should be cautious in drawing any final conclusions about these reported findings. In fact, they have now been challenged (Treisman, Squire, \& Green, 1974; Wardlaw \& Kroll, 1976; Bowers \& Brenneman, Note 1).

Even though we lean toward an interpretation of our results in terms of a failure of form perception simultaneous with successful perception of other properties of the object, we cannot entirely rule out the alternative interpretation that inattention leads not to a failure of form perception but to a failure of memory. According to this view, the shape of the unattended figure is perceived no differently than the attended one. The argument would be that for certain reasons, perceptions that occur without benefit of attention either are not transferred into memory at all or, if they are, are subject to rapid decay. As to the latter, one might argue that by the time of the test in Experiment 1 , there is no basis in memory for recognition of the shape of the unattended figures. It is unlikely, however, that the delay and/or interference based on the exposure of the 10 slides is responsible for the poor recognition performance. In the previous method of achieving inattention (Rock et al., 1976), only one figure was exposed, and the test came directly afterward, but the results were similar. Recognition based on form was at a chance level. Moreover, in the present research, Experiment 4 isolated one figure on the basis of potential familiarity and tested for recognition immediately afterward. The results again showed an absence of recognition.

It could be argued that in this experiment using a familiar figure, it was not form perception that suffered but recognition at the time of exposure. In other words it could be maintained that the form of the familiar 
objects qua form was perceived but that inattention for some reason blocked access to memory. That is a logical possibility, but ordinarily we assume that recognition is based on similarity of perceived to remembered attributes and does not require intention. Thus, if perception occurred, recognition should also have occurred. In any event, the argument would then have to maintain that this perceived but unrecognized form did not establish a viable memory. For if it did, one would think it would have been elicited in the period immediately afterward when the experimenter probed for such memory including the re-representation of the figure.

We, therefore, tend to discount the possibility that memories of the unattended figures are established but do not endure. As to the possibility that such memories are not established, the question arises as to why this might be the case. One explanation of why an unattended item might be perceived but not transferred into memory is that the requirements of the attention task (e.g., shadowing) preempt or prevent the processing requirements for such transfer. Although this is an argument that is relevant to our present findings, in which such an attention task is utilized, it is questionable whether it is applicable to the method previously used (Rock et al., 1976), in which only one item at a time is presented and in which there is no other item to which to attend. In any event, we wonder if the assertion that a memory is not established is a different theoretical statement than the assertion that there is a failure of perception. For if any processing of a figure occurs beyond that which underlies iconic representation, it would presumably lead to a perception and to memory formation. Otherwise expressed, it is not clear what it means to say that some further processing other than that which establishes a percept must occur to enter that percept into memory.

Finally, the results of Experiment 5 demonstrate that in fact various features of the unattended object are both perceived and retained in memory. Thus, to maintain that the unattended object's shape is perceived but either does not establish a memory or does but it is one that does not endure re- quires the assumption that everything about the object is perceived but only certain of its aspects enter memory or are remembered.

All things considered, then, it would seem to be more parsimonious, more plausible, and in keeping with much that we know about cognition to assume that failure to encode the shape into memory is based on a failure in the act of perception. One reason why some may find our conclusion difficult to accept is that in those cases in daily life in which at a given moment we are not attending to a pattern at which we are looking, there is the distinct impression nonetheless that something is there and that it has certain phenomenal characteristics. Two factors may be behind this impression, both compatible with our conclusion: One is that by virtue of the iconic representation, we as observers realize that the potential to transmute this impression into a distinct shape is there. Another, based on the findings of our last experiment, is that certain properties other than shape per se are perceived (and, therefore, stored) even without focal attention.

\section{Reference Note}

1. Bowers, K. S., \& Brenneman, H. A. Unattended information and hypnotic susceptibility. Paper presented at the convention of the Society for Clinical and Experimental Hypnosis, Montreal, Canada, October 1974.

\section{References}

Bregman, A.S. Perception and behavior as composition of ideals. Cognitive Psychology, 1977, 9, 250-292.

Broadbent, D. E. Perception and communication. New York: Pergamon Press, 1958.

Cherry, E. C. Some experiments on the recognition of speech with one and with two ears. Journal of the Acoustical Society of America, 1953, 25, 975-979.

Corteen, R. S., \& Wood, B. Autonomic responses to shock-associated words in unattended channel. Journal of Experimental Psychology, 1972, 94, 308-313.

Eriksen, C. W., \& Lappin, J. S. Selective attention and very short-term recognition memory for nonsense forms. Journal of Experimental Psychology, 1967, $73,358-364$.

Hochberg, I. Attention, organization, and consciousness. In D. Mostophy (Ed.), Attention: Contemporary theory and analysis. New York: Appleton Century, 1970.

Lewis, J. L. Semantic processing of unattended messages using dichotic listening. Journal of Experimental Psychology, 1970, 85, 225-228. 
Moray, N. Attention in dichotic listening: Affective cues and the influence of instructions. Quarterly Journal of Experimental Psychology, 1959, 9, 56-60.

Neisser, U. Cognitive psychology. New York: Appleton, 1967

Reed, S. D. Structural descriptions and the limitations of visual images. Memory \& Cognition, 1974, 2, 329 336.

Rock, I. Orientation and form. New York: Academic Press, 1973.

Rock, I. An introduction to perception. New York: Macmillan, 1975.

Rock, I., \& Gilchrist, A. Induced form. American Journal of Psychology, 1975, 88, 475-482.

Rock, I., \& Halper, F. Form perception without a retinal image. American Journal of Psychology, 1969, 82, 425-440.

Rock, I., Halper, F., \& Clayton, T. The perception and recognition of complex figures. Cognitive Psychology, $1972,3,655-673$.

Rock, I., Schauer, R., \& Halper, F. Form perception without attention. Quarterly Journal of Experimental Psychology, 1976, 28, 429-440.
Rock, I., \& Sigman, E. Intelligence factors in the perception of form through a moving slit. Perception, 1973, 2, 357-369.

Shiffrin, R. M., \& Schneider, W. Controlled and automatic information processing: II. Perceptual learning, automatic attending, and a general theory. Psychological Review, 1977, 84, 129-190.

Sperling, G. The information available in brief visual presentations. Psychological Monographs, 1960, 74(11, Whole No. 498).

Treisman, A. M. Strategies and models of selective attention. Psychological Review, 1969, 76, 282-299.

Treisman, A. M., Squire, R., \& Green, J. Semantic processing in dichotic listening? A replication. $\mathrm{Mem}$ ory and Cognition, 1974, 2, 641-646.

Wardlaw, K. A., \& Kroll, N. A. E. Autonomic responses to shock-associated words in a nonattended message: A failure to replicate. Journal of Experimental Psychology: Human Perception and Performance, 1976, 2, 357-360.

Received November 2, 1979 